\title{
Ruptura septal de localización atípica posterior a infarto del miocardio. Caso clínico
}

\author{
A septal rupture of atypical location after myocardial infarction. A clinical case
}

Diego Incontri-Abraham¹, Juan J. Juárez-Vignon-Whaley ${ }^{1}$ Pablo J. González-Velásquez², Alondra N. Flores-García ${ }^{3}$, Nilda Espinola-Zavaleta ${ }^{3 *}$

${ }^{1}$ Facultad de Ciencias de la Salud, Universidad Anáhuac México Norte; ${ }^{2}$ Departamento de Enseñanza Médica, Instituto Nacional de Cardiología Ignacio Chávez; ${ }^{3}$ Departamento de Cardiología Nuclear, Instituto Nacional de Cardiología Ignacio Chávez. Ciudad de México, México.

Paciente masculino de 46 años de edad con antecedentes personales de hipertensión arterial sistémica, tabaquismo y etilismo y heredofamiliares de hipertensión arterial sistémica. El padecimiento inició con cuadro de astenia, adinamia, disnea progresiva, edema de miembros inferiores y aumento del volumen abdominal, por lo que acudió con el médico, quien decidió hospitalizarlo. El paciente recibió tratamiento médico con captopril, furosemida y espironolactona, sin mejoría de los síntomas, motivo por el cual fue referido a la institución. Al llegar al servicio de urgencias, el sujeto se encontraba estable. A la exploración física estaba consciente y orientado, con adecuada coloración e hidratación de tegumentos, ingurgitación yugular grado Il y pulsos carotídeos simétricos de adecuada amplitud. La auscultación reveló estertores crepitantes en la región subescapular bilateral, hipoventilación en la región subescapular derecha y síndrome de derrame ipsilateral. El choque de la punta se palpó en el sexto espacio intercostal, línea media clavicular izquierda y se detectó frémito paraesternal izquierdo. Los ruidos cardíacos eran rítmicos, con S3 y soplo holosistólico en mesocardio III/IV, con irradiación excéntrica y sin frote pericárdico. Las extremidades inferiores estaban íntegras, simétricas, con edema hasta el tercio proximal tibial bilateral y llenado capilar inmediato. Los pulsos eran simétricos y de adecuada amplitud en las cuatro extremidades. El electrocardiograma mostró ritmo sinusal con FC de $83 \mathrm{lpm}$, dextrorrotación, crecimiento de la aurícula izquierda e imagen de infarto reciente inferolateral, con onda q, lesión subepicardica e inversión simétrica de la onda T en DII, III, aVF, V5 y V6 (Fig. 1). En la radiografía de tórax se observó cardiomegalia grado Il con hipertensión venocapilar pulmonar (Fig. 2). El ecocardiograma demostró ruptura septal apical de $17 \mathrm{~mm}$ (Fig. 3), con Qp/Qs de 3.6 y gradiente a través del defecto de $26 \mathrm{mmHg}$ y presión sistólica de arteria pulmonar de 74 mmHg (Fig. 4), dilatación auricular izquierda y función sistólica del ventrículo izquierdo normal, con fracción de expulsión de 58\%. El paciente ingresó a la sala de hemodinámica para colocación de un balón intraaórtico de contrapulsación (BIAC). Se realizó coronariografía diagnóstica, que demostró tron$\mathrm{co}$, arteria descendente anterior izquierda y circunfleja sin lesiones angiográficas significativas, con flujo distal TIMI 3. La arteria coronaria derecha dominante mostraba lesión tubular ulcerada en el segmento medio, de bordes mal definidos, excéntrica, con sitio de máxima estenosis del $70 \%$ y oclusión total crónica de la descendente posterior en el segmento medio (flujo TIMI 0), con circulación colateral de la arteria descendente anterior, Rentrop 3 (Fig. 5). En la ventriculografía se

\section{Correspondencia:}

*Nilda Espinola-Zavaleta

E-mail: niesza2001 @ hotmail.com
Fecha de recepción: 10-02-2019

Fecha de aceptación: 27-06-2019

DOI: $10.24875 /$ ACM.19000064
Disponible en internet: 13-09-2019 Arch Cardiol Mex. 2021;91(1):130-134

www.archivoscardiologia.com

1405-9940 @ 2019 Instituto Nacional de Cardiología Ignacio Chávez. Publicado por Permanyer México SA de CV. Este es un artículo Open Access bajo la licencia CC BY-NC-ND (http://creativecommons.org/licenses/by-nc-nd/4.0/). 


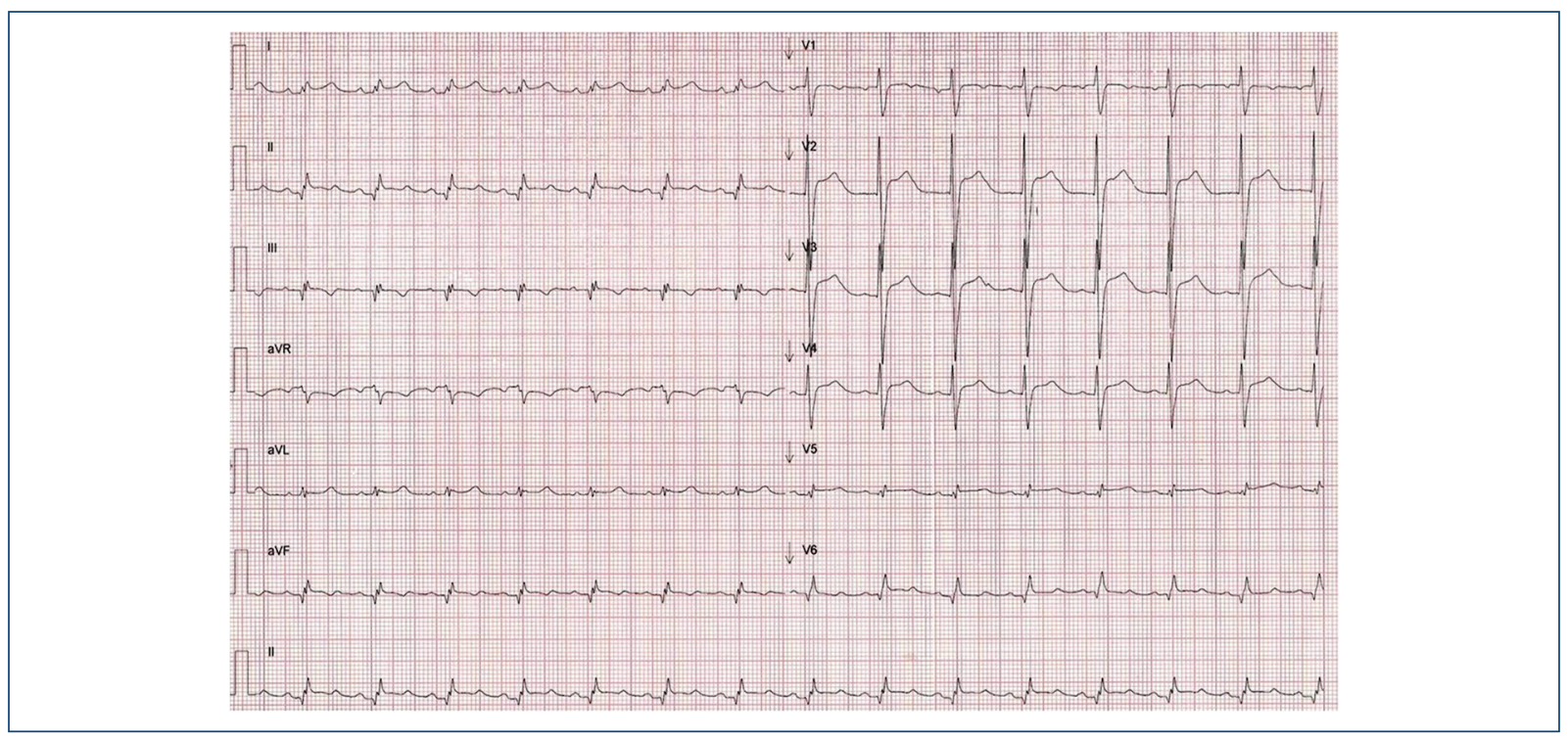

Figura 1. Electrocardiograma en ritmo sinusal con FC de 83 Ipm, dextrorrotación, crecimiento de la aurícula izquierda y una imagen de infarto reciente inferolateral, con onda $q$, lesión subepicardica e inversión simétrica de la onda T en DII, III, aVF, V5 y V6.

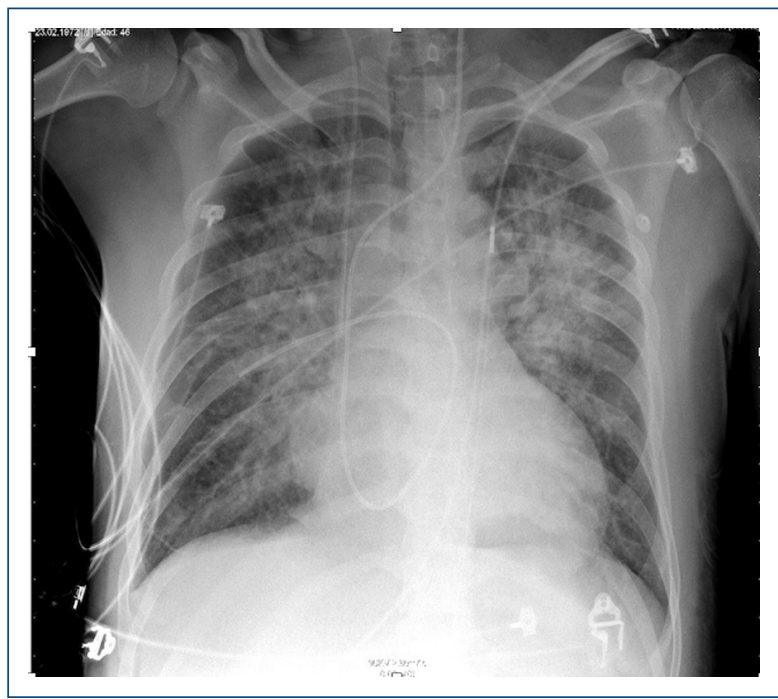

Figura 2. Radiografía de tórax que revela cardiomegalia grado II, hipertensión venocapilar pulmonar considerable, catéter de flotación en la arteria pulmonar y balón de contrapulsación aórtico.

identificó una comunicación interventricular apical y la presión teledistólica del ventrículo izquierdo fue de $35 \mathrm{mmHg}$. Se inició tratamiento con diurético de asa, nitroprusiato de sodio y levosimendán a dosis-respuesta, con evidente mejoría. Se colocó un catéter de Swan-Ganz, que demostró una presión capilar pulmonar de $34 \mathrm{mmHg}$.
Tras la estabilización hemodinámica, se le realizó una intervención de cierre de la comunicación interventricular (CIV) con parche de pericardio bovino y colocación de hemoducto venoso a la descendente posterior.

En su evolución, el paciente cursó con un cuadro de sepsis de origen pulmonar, debido al desarrollo de una neumonía relacionada con la ventilación mecánica. Desarrolló deterioro hemodinámico a pesar de la administración de vasopresores a dosis altas, signos de hipoperfusión persistente con gasto cardíaco de $1.5 \mathrm{~L} / \mathrm{min}$, hipotensión arterial de $80 / 40 \mathrm{mmHg}$ y acidosis láctica. El ecocardiograma transtorácico evidenció la presencia de taponamiento cardíaco con separación de las hojas del pericardio de $2.1 \mathrm{~cm}$, colapso de cavidades derechas e incremento de la variabilidad del flujo transmitral y transtricuspídeo. Se creó una ventana pericárdica urgente con extracción de $140 \mathrm{ml}$ de material hemático. El paciente falleció ese mismo día y se establecieron los diagnósticos de choque séptico, choque cardiogénico, neumonía bacteriana e infarto agudo del miocardio (IAM).

A pesar de los recientes avances en el diagnóstico y tratamiento de la cardiopatía isquémica, ésta es aún la principal causa de morbimortalidad en el mundo. Incluso los pacientes que sobreviven al IAM tienen riesgo de sufrir complicaciones mecánicas que disminuyen en grado significativo la sobrevida a corto y largo plazos; más del $50 \%$ de las muertes ocurren 


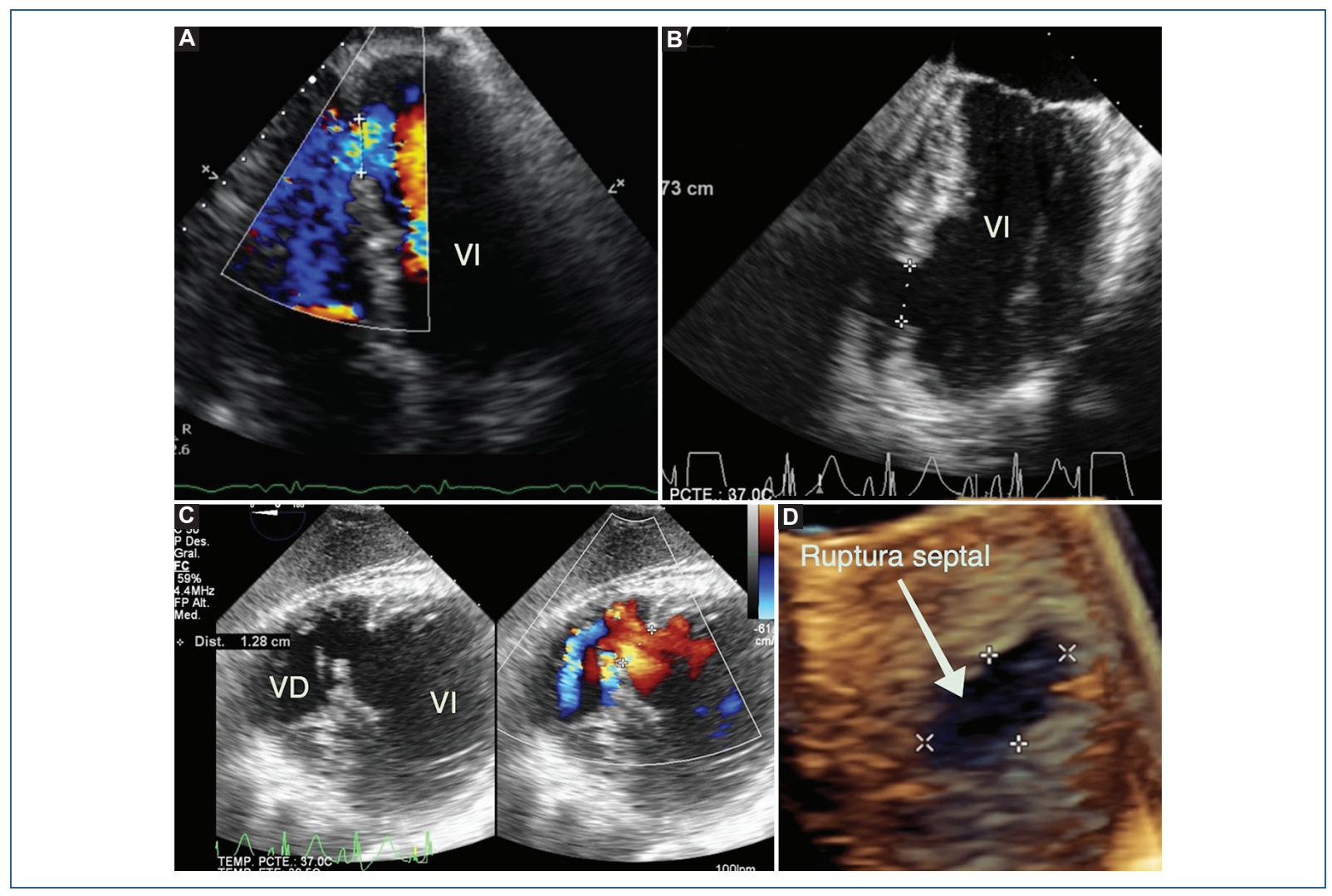

Figura 3. A: Ecocardiograma transtorácico bidimensional y con Doppler a color en el plano apical de cuatro cámaras, que muestra ruptura septal en la porción apical del septum. B: Ecocardiograma transesofágico bidimensional que muestra la ruptura septal en la porción apical del septum. C: Ecocardiograma transgástrico bidimensional y con Doppler a color, a nivel de ambos ventrículos, que muestra la ruptura septal. D: Reconstrucción tridimensional de la ruptura septal que tiene forma ovalada (flecha blanca).

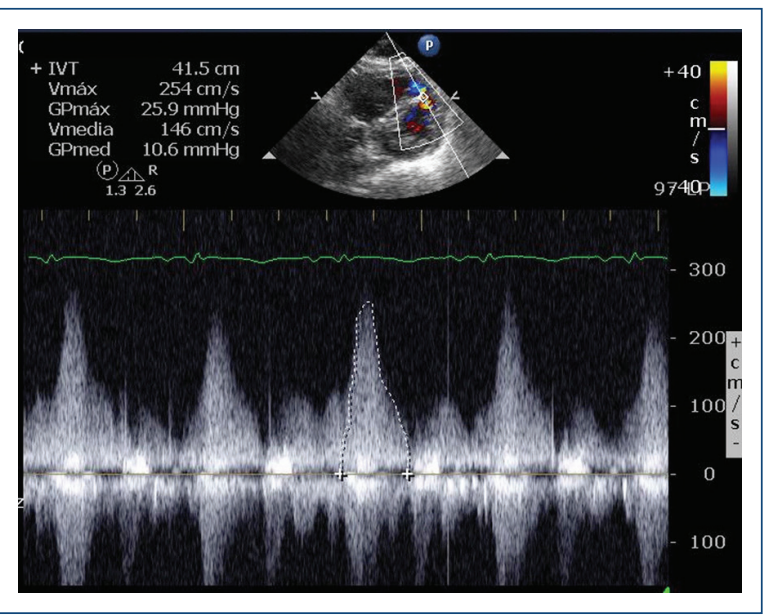

Figura 4. Ecocardiograma transtorácico bidimensional y con Doppler a color que muestra ruptura septal apical; el gradiente a través de la comunicación interventricular (27 $\mathrm{mmHg}$ ) se calculó con Doppler continuo. La presión sistólica de la arteria pulmonar (PSAP) se calculó con la siguiente fórmula: presión sistólica sistémica-gradiente transCIV $=100-26=74 \mathrm{mmHg}$. dentro de los primeros 28 días posteriores al IAM, sin que el paciente llegue al hospital. El resto muere en las primeras 24 horas de hospitalización $n^{1,2}$.

Con el advenimiento de la intervención coronaria percutánea (ICP), la incidencia de las complicaciones mecánicas del IAM ha disminuido de manera significativa en los últimos años ${ }^{3}$ y en la actualidad es < $1 \%$. Sin embargo, aún representan una emergencia clínica y son una importante causa de mortalidad a corto plazo 4 .

Las complicaciones del IAM pueden ser mecánicas, eléctricas, inflamatorias, isquémicas y embólicas (Tabla 1$)^{4}$ y su aparición eleva la mortalidad, en particular en los casos sin tratamiento de reperfusión o con trombólisis tardía ${ }^{5}$. El choque cardiogénico es la complicación mecánica más frecuente y la principal causa de muerte después de un $\mathrm{IAM}^{3}$.

La incidencia de ruptura septal antes de la reperfusión ${ }^{6}$ era de 1 a 3\%, y con la trombólisis y la ICP ha descendido a 0.17 a $0.31 \%{ }^{7}$. En la actualidad, esta 


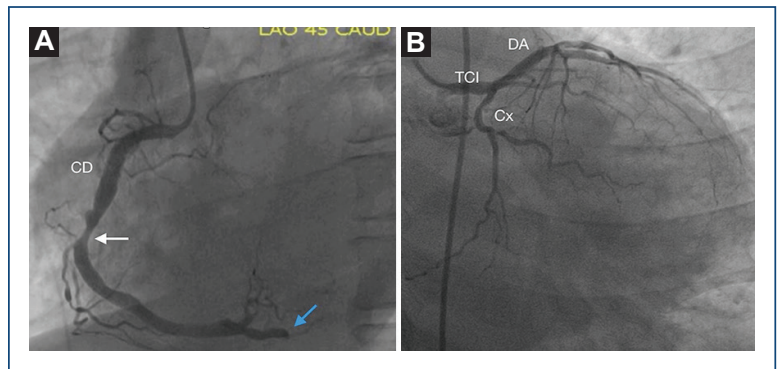

Figura 5. Coronariografía en la proyección oblicua anterior izquierda a $45^{\circ}$. A: Coronaria derecha dominante con lesión tubular ulcerada en el segmento medio, de bordes mal definidos, excéntrica, con sitio de máxima estenosis del $70 \%$ (flecha blanca) y oclusión total crónica de la descendente posterior en el segmento medio, flujo TIMI 0 (flecha azul), con circulación colateral de la arteria descendente anterior, Rentrop 3. B: En la proyección oblicua anterior derecha se observa el tronco de la coronaria izquierda sin lesiones angiográficas significativas, I-a arteria circunfleja y la descendente anterior izquierda sin obstrucciones angiográficas significativas con flujo distal TIMI 3.

CD: coronaria derecha; $C x$ : circunfleja; DA: descendente anterior; TCl: tronco de la coronaria izquierda.

Tabla 1. Complicaciones del infarto agudo del miocardio

\begin{tabular}{|l|l|}
\hline Complicaciones & Subtipos \\
\hline Mecánicas & $\begin{array}{l}\text { Choque cardiogénico, ruptura de pared } \\
\text { libre, ruptura del septum ventricular, } \\
\text { aneurisma ventricular e insuficiencia } \\
\text { mitral aguda }\end{array}$ \\
\hline Eléctricas & $\begin{array}{l}\text { Arritmias, bloqueos AV y bloqueos } \\
\text { fasciculares }\end{array}$ \\
\hline Inflamatorias & Síndrome de Dressler \\
\hline Isquémicas & Angina posterior a infarto \\
\hline Embólicas & Trombosis mural y embolismo sistémico \\
\hline
\end{tabular}

Modificada de Moreno et al., $2017^{4}$.

complicación mecánica representa sólo el 5\% de todas las muertes por IAM $^{3,4,8}$, pero cuando se presenta tiene una mortalidad hasta del $46 \%$ en la primera semana y alcanza el $90 \%$ en los dos primeros meses en aquellos pacientes no sometidos a tratamiento quirúrgico $0^{9,10}$. El paciente fue sometido a revascularización y cierre de CIV, pero falleció en los dos primeros meses tras el inicio del padecimiento. La bibliografía registra que aun con la reparación quirúrgica, la mortalidad en estos enfermos es de $40 \%{ }^{7,11}$.
Las manifestaciones clínicas de la ruptura septal interventricular ocurren en los primeros tres a cinco días después del IAM, aunque algunos informes mencionan que pueden presentarse incluso hasta en los 30 días posteriores al infarto ${ }^{12}$. Este caso que no recibió tratamiento de reperfusión mostró ruptura septal cuatro días después del episodio isquémico. Entre los factores de riesgo que predisponen a la ruptura septal destacan la hipertensión, edad avanzada, sexo femenino, ausencia de angina, tiempo de isquemia prolongado, ausencia de colaterales, IAM anterior e IAM extenso $0^{1,13}$. Este paciente era hipertenso, pero el factor de riesgo más significativo para la ruptura septal fue el tiempo prolongado de isquemia sin reperfusión.

Cabe destacar que en los infartos de localización anterior, la ruptura septal es más frecuente en la región apical, mientras que en los infartos de la cara inferior o lateral aparecen en la región posterobasal|,14-16. En contraste con estos hallazgos, este paciente sufrió un IAM transmural posteroinferior con ruptura septal en la porción apical, presentación rara de esta complicación. Hay que recordar que la mayor parte del septum ventricular (2/3) está irrigada por la coronaria izquierda, pero en un tercio por la coronaria derecha. Esto podría explicar la inusual presentación de ruptura septal apical en presencia de obstrucción de la arteria descendente posterior.

En más de $70 \%$ de los pacientes, la presentación clínica más frecuente corresponde al colapso hemodinámico y soplo holosistólico ${ }^{17-19}$. El ecocardiograma transtorácico es el estudio diagnóstico de elección para las complicaciones mecánicas del $\mathrm{IAM}^{18}$, aunque en ocasiones el ecocardiograma transesofágico, especialmente la técnica tridimensional, es superior para delinear con precisión la extensión completa de la ruptura ${ }^{16}$.

Si bien las guías de la ACC/AHA recomiendan que la ruptura septal ventricular posterior a infarto exige intervención urgente ${ }^{20}$, algunos estudios muestran que la mortalidad del tratamiento quirúrgico es mayor cuanto más temprana sea la intervención ${ }^{8}$. Los pacientes que entran a quirófano en los primeros siete días poseen una mortalidad de $54.1 \%$, en comparación con el $18.4 \%$ de aquéllos operados después de siete días. La mortalidad es mayor al $60 \%$ si el procedimiento se practica en las primeras 24 horas $^{6}$. Debido a ello, el uso del BIAC, los fármacos inotrópicos, vasodilatadores y los métodos invasivos son indispensables en la estabilización prequirúrgica del paciente 1 . La mortalidad quirúrgica en los casos con choque cardiogénico es de 20 a $50 \%{ }^{15}$. 
La incidencia de derrame pericárdico, hemodinámicamente significativo, se ha descrito en 1 a $2 \%$ de todas las operaciones cardíacas; la presencia de lesión renal y el tiempo en bomba de circulación extracorpórea prolongado son factores de riesgo independientes para el desarrollo de esta complicacion ${ }^{21}$, ambas alteraciones presentes en este paciente. Aunque los autores consideran que el taponamiento cardíaco no fue la causa de muerte en este paciente, sí contribuyó al deterioro hemodinámico y por consiguiente a la hipoperfusión tisular sostenida.

El cierre percutáneo de la ruptura septal es una alternativa que ha mostrado resultados favorables a corto y largo plazo ${ }^{1,3,18,20}$; en este caso, los bordes del defecto no eran adecuados para la fijación de un dispositivo de cierre percutáneo.

\section{Conclusión}

Este caso es de especial interés porque presentó un infarto transmural de la pared posteroinferior del ventrículo izquierdo, que presentó ruptura septal en la porción apical del septum, localización atípica de esta complicación diagnosticada mediante los datos clínicos, la exploración física y la ecocardiografía. A pesar de las intervenciones realizadas, el paciente murió por choque cardiogénico.

\section{Financiamiento}

No se recibió ningún financiamiento.

\section{Conflicto de intereses}

Los autores no tienen ningún conflicto de intereses que declarar.

\section{Responsabilidades éticas}

Protección de personas y animales. Los autores declaran que para esta investigación no se han realizado experimentos en seres humanos ni en animales.

Confidencialidad de los datos. Los autores declaran que han seguido los protocolos de su centro de trabajo sobre la publicación de datos de pacientes.

Derecho a la privacidad y consentimiento informado. Los autores han obtenido el consentimiento informado de los pacientes y/o sujetos referidos en el artículo. Este documento obra en poder del autor de correspondencia.

\section{Bibliografía}

1. Caballero-Borrego J, Hernández-García JM, Sanchís-Fores J. Complicaciones mecánicas en el infarto agudo de miocardio. ¿Cuáles son, cuál es su tratamiento y qué papel tiene el intervencionismo percutáneo? Rev Esp Cardiol. 2009;9:62-70.

2. Magalhães $P$, Mateus $P$, Carvalho $S$, Leão $S$. Relationship between treatment delay and type of reperfusion therapy and mechanical complications of acute myocardial infarction. Eur Heart J Acute Cardiovasc Care. 2016;5(5):468:74

3. Bajaj A, Sethi A, Rathor P, Suppogu N, Sethei A. Acute complications of myocardial infarction in the current era: diagnosis and management. J Investig Med. 2015;63(7):844-56.

4. Moreno JQ, Ariza Rodríguez DJ, Rugeles T, Bermúdez López LM. Complicaciones mecánicas del infarto agudo de miocardio: aunque infrecuentes, potencialmente letales. Sociedad Colombiana de Cardiología y Cirugía Cardiovascular. Rev Colomb Cardiol. 2017;24(5):505-9. Available from: http://dx.doi.org/10.1016/j.rccar.2017.04.005

5. Nakatani D, Sato H, Kinjo K, Mizuno H, Hishida E, Hirayama A, et al. Effect of successful late reperfusion by primary coronary angioplasty on mechanical complications of acute myocardial infarction. Am J Cardiol. 2003;92(03):785-8.

6. Jones BM, Kapadia SR, Smedira NG, Robich M, Tuzcu EM, Menon V et al. Clinical update ventricular septal rupture complicating acute myocardial infarction: a contemporary review. Eur Heart J. 2014;1-9.

7. French JK, Hellkamp AS, Armstrong PW, Cohen E, Kleiman NS, Connor CMO, et al. mechanical complications after percutaneous coronary intervention in ST-Elevation Myocardial Infarction. Am J Cardiol. 2010;105(1):59-63. Available from: http://dx.doi.org/10.1016/j.amjcard.2009.08.653

8. Liebelt JJ, Yang Y, Derose JJ, Taub CC. Ventricular septal rupture complicating acute myocardial infarction in the modern era with mechanical circulatory support: a single center observational study. Am J Cardiovasc Dis. 2016;6(1):10-6.

9. Birnbaum Y, Fishbein M, Blanche C, Siegel RJ. Ventricular septal rupture after acute myocardial infarction. N Engl J Med. 2002;347(18):1426-32.

10. Premchand RK, Garipalli R, Padmanabhan TNC, Manik G. Percutaneous closure of post-myocardial infarction ventricular septal rupture - A single centre experience. Indian Heart J. 2017;69(December 2005):S24-7. Available from: http://dx.doi.org/10.1016/j.ihj.2016.10.004

11. Cho JH, Sattiraju S, Mehta S, Missov E. Delayed ventricular septal rupture complicating acute inferior wall myocardial infarction. BMC Res Notes [Internet]. BMC Research Notes; 2013;6(1):1. Available from: BMC Research Notes

12. Yip H, Fang $\mathrm{C}$, Tsai $\mathrm{K}$, Chang $\mathrm{H}$, Yeh $\mathrm{K}$, Fu M, et al. The potential impact of primary percutaneous coronary intervention on ventricular septal rupture complicating acute ymocardial infarction. Chest. 2003;125(5):16228. Available from: http://dx.doi.org/10.1378/chest.125.5.1622

13. Serpytis P, Karvelyte N, Serpytis R, Kalinauskas G, Rucinskas K, Samalavicius $R$, et al. Post-Infarction ventricular septal defect: risk ractors and early outcomes. Hell J Cardiol. 2015;56:66-71.

14. Hayashi T, Hirano Y, Takai H, Kimura A, Taniguchi M, Kurooka A, et al. Usefulness of ST-segment elevation in the inferior leads in predicting ventricular septal rupture in patients with anterior wall acute myocardial infarction. Am J Cardiol. 2005;96(8):1037-41.

15. González Rosas IA, Hernández Santamaría I, Velasco AV, Pérez Salgado H, López Gómez LM, García Aguilar BS, et al. Complicaciones mecánicas del infarto: rotura de pared libre ventricular. Presentación de un caso. Rev Mex Cardiol. 2014;25(1):36-42.

16. Reeder GS. Concise review for primary-care physicians identification and treatment of complications of myocardial infarction. Mayo Clin Proc. 1995;70(9):880-4. Available from: http://dx.doi.org/10.4065/70.9.880

17. Su W, Wang S, Wang J, Zhang J, Chen Y. Acute myocardial infarction complicated with ventricular septal rupture: report of three cases. Cardiol Res. 2013;4(151):203-6.

18. Wilansky S, Moreno CA, Lester SJ. Complications of myocardial infarction. Crit Care Med. 2007;35(8):348-54

19. Lavie CJ, Gersh BJ, Ch B, Phil D. Mechanical and electrical complications of acute myocardial infarction. Mayo Clin Proc. 1990;65:709-30.

20. Tang L, Fang Z, Hu X, Tang J, Shen X, Lu X, et al. Non-surgical repair of ventricular septal rupture after acute myocardial infarction. Int $\mathrm{J}$ Cardiol. 2015;185:328-32. Available from: http://dx.doi.org/10.1016/j.jjcard.2015.03.144

21. Ashikhmina E, Schaff H, Sinak L, Li Z, Dearani J, et al. Pericardial effusion after cardiac surgery: risk factors, patient profiles, and contemporary management. Ann Thorac Surg. 2010;89:112-8. 